\title{
The Impact of Spume Droplets and Wave Stress Parameterizations on Simulated Near-Surface Maritime Wind and Temperature
}

\author{
VALDIR INNOCENTINI AND IURY ANGELO GONÇALVES \\ Instituto Nacional de Pesquisas Espaciais, São José dos Campos, São Paulo, Brazil
}

(Manuscript received 31 August 2009, in final form 19 January 2010)

\begin{abstract}
The influence of ocean gravity waves on the wind and temperature above the surface is investigated using a one-dimensional boundary layer model. The effect of the wave-induced stress is evaluated using three parameterizations: wave age ( $\mathrm{WaAg}$ ), wave steepness (WaSt), and wind action on the wave spectrum (WiAc). It is found that while the WaAg is more effective in reducing the wind for young waves, in the WaSt approach the maximum reduction is for old waves. On the other hand, the WiAc is very sensitive to the energy present in high frequencies corresponding to periods less than $2 \mathrm{~s}$, which are found in both young and mature spectra. Since observations show that most of the wave stress is due to the small-period wave energy, in this aspect the WaSt parameterization is not recommended; WaAg is not as accurate; and thus WiAc is the best among the three, although its computational cost is the highest.

The droplet load contribution to the total surface stress can be neglected for the droplet spectrum produced by $10-\mathrm{m}$ wind speeds up to $15 \mathrm{~m} \mathrm{~s}^{-1}$, but its importance increases with the speed and its magnitude becomes about $1 / 5$ of the total stress for wind speed $\sim 30 \mathrm{~m} \mathrm{~s}^{-1}$.

Concerning the latent and sensible heat fluxes accompanying the production of spume droplets by waves, a feasible microphysical formulation for operational use in weather forecasting models is proposed. The droplet spectrum is assumed to be a product of two functions, one depending on the windsea Reynolds number and the other on the droplet radius spectrum. The bulk effect of the latter is analytically evaluated and stored in a table as a function of air temperature $T_{a}$, relative humidity $R_{\%}$, and significant wave height $H_{s}$. In numerical experiments with initial sea surface temperature $5 \mathrm{~K}$ higher than the air surface, latent and sensible heat contributions to the air temperature are computed as a function of the wave spectra. The launched droplet spectrum (which increases the air temperature due to sensible heat) and the relative humidity (which controls the cooling due to the droplet evaporation) define the heat budget and the air temperature evolution. Although in these experiments the sea temperature is much higher than the air temperature, the results show a noticeable dominance of the evaporative cooling in the lower atmosphere mainly for smaller significant wave height. Some air warming is noticeable only from a threshold around $H_{s} \geq 5 \mathrm{~m}$.
\end{abstract}

\section{Introduction}

In recent years the inclusion of sea surface waveinduced stress and sea spray droplets has been recognized as a crucial requirement to quantify accurately the exchange of heat, momentum, and moisture between the air and the ocean, mainly in studies of severe meteorological events. However, the full microphysics of droplet evolution demands a great deal of computer time in numerical simulations. To overcome this difficulty and avoid the need for a detailed description, Fairall et al. (1994)

Corresponding author address: Dr. Valdir Innocentini, INPE, Av. dos Astronautas, 1756, São José dos Campos, São Paulo, Brazil. E-mail: valdir@cptec.inpe.br and Andreas (2005) introduced approximate formulas. Although the Andreas approach deals explicitly with the droplets, Fairall et al. simplify this treatment but overestimate the latent heat released. One objective of the present research is to propose a bulk formulation of microphysical mechanisms due to the sea spray based on Andreas's simplifications. The majority of the procedures adopted were developed by Andreas and collaborators but are repeated here for completeness and to emphasize the approximations proposed.

During wave breaking, air is entrained into the water, producing bubbles that burst upon emerging at the sea surface, ejecting droplets into the air. They can be classified into film and jet droplets, the first produced when the bubble bursts and the second by water jetting out of 
the bubble cavity after the burst. A third type, named spume, is produced by wind tearing of the wave crests. The radii of the droplet classes range from 0.1 to $50 \mu \mathrm{m}, 1$ to $100 \mu \mathrm{m}$, and 20 to $500 \mu \mathrm{m}$, respectively (Andreas 2002).

Zhao et al. (2006) described the various conflicting conclusions about the importance of sea spray droplets in the several air-sea exchange processes. For example, while $\mathrm{Wu}(1998)$ concluded that less than $3 \%$ of the sea evaporation comes from droplets, Andreas (2004) argued that the surface stress due to the spray with the $10-\mathrm{m}$ height wind speed $U_{10}$ equal to $30 \mathrm{~m} \mathrm{~s}^{-1}$ is about $10 \%$ of the total, but, when the wind is nearly $60 \mathrm{~m} \mathrm{~s}^{-1}$, all of the stress is due to spray. Zhao et al. attributed much of the variability in these estimates to the disparate nature of the published sea spray generation functions (SSGF). Andreas (2002) reported a range of six orders of magnitude in 13 SSGF described in the published research. Certainly, such great discrepancies are due to the difficulty of obtaining measurements. Although the quantity of smaller droplets generated by the film and jet is greater, spume droplets with radius between 30 and $500 \mu \mathrm{m}$ are the most relevant for air-sea interactions and deserve special attention.

After a spume droplet has been introduced into the atmosphere, its trajectory will be determined by its initial velocity, atmospheric turbulence, and gravitational force until it falls back to the sea. During the airborne phase the droplet (i) extracts momentum if the sea and air speeds are different, (ii) transfers sensible heat if the sea and air temperatures are different, and (iii) transfers latent and sensible heat if the droplet evaporation layer (DEL) is not saturated.

During the latent heat transfer, the droplet radius is reduced from the initial $r_{i}$ to an equilibrium radius $r_{\mathrm{eq}}$. However, in general, for large spume droplets, their residence time within the evaporation layer is short and their radius is only reduced to $r_{f}>r_{\mathrm{eq}}$. Therefore, the residence time in the atmosphere of each element of the launched droplet collection must be known, and this value depends on the droplet terminal velocity and the height at which it is launched into the atmosphere. Andreas and Emanuel (2001) observe that the effect of reentrance of droplets into the sea without evaporating has a significant role in the budget of enthalpy necessary to sustain tropical cyclones.

Regarding the wave-induced and droplet load stresses, both are considered in atmospheric models to account for the momentum and temperature profiles. The wave motion affects the roughness length, and the droplets during their residence time represent an extra load to the air, so the surface stress is modified.

The inclusion of so many details in sea-air parameterizations can consume a great deal of computer time in numerical simulations. We have two main objectives in this research: (i) to present feasible approximations for a bulk parameterization of spume droplets to be implemented in a coupled system composed of wave and atmospheric numerical models and (ii) to study the impact of the wave motion and the spume droplets on the nearsurface maritime wind and temperature. Unlike other studies, where the effects of latent and sensible heat sources due to the droplets on the intensification of a tropical cyclone are investigated, here we focus on the impact of a wave field on the atmospheric profiles of momentum and temperature initially in steady state. The experiments consist of imposing a wave field independent of local wind, covering a large domain of peak frequencies from young to old waves. The effect of several approaches depending on the wave stages is discussed and not only with a fully developed wave spectrum determined by the local wind as adopted in some studies (e.g., Fairall et al. 1994).

Section 2 describes the one-dimensional atmospheric model employed to simulate the atmospheric profile evolution. Section 3 presents the spray parameterization. Three wave-effect parameterizations commonly adopted in numerical models are presented in section 4: wave age (WaAg), wave steepness (WaSt), and input of energy into the wave spectrum [wind action (WiAc)]. Section 5 discusses the numerical simulations and the impact of these wave stress parameterizations and the spume droplets on the near-surface wind and temperature. Finally, section 6 summarizes the main findings.

\section{The one-dimensional atmospheric model}

Since the main focus of this research is on the evolution of marine profiles under the effects of surface waves and sea spray produced by breaking waves, we are proposing a boundary layer atmospheric model with a simple dynamic formulation. Two methods are frequently employed in numerical models to introduce the effect of the waves on the atmosphere: the roughness length and the wave-induced stress. In the first, the roughness depends on wave parameters while, in the second, the surface stress is separated into wind, wave-induced, and water load stress; in this case, the wave-induced stress is obtained from the wave spectrum.

\section{a. Governing equations}

The numerical model is composed of prognostic equations for the horizontal momentum $u$, potential temperature $\theta$, and water vapor mixing ratio $q$. If $X$ represents a generic prognostic variable, its time derivative is 


$$
\frac{\partial X}{\partial t}=\frac{\partial \tau}{\partial z}+R_{X}
$$

where $R_{X}$ represents all terms except the vertical derivative of the turbulent vertical flux $\tau, t$ is the time, and $z$ is the vertical axis. Equation (2.1) can be discretized for a level $i$,

$$
\frac{\partial X_{i}}{\partial t}=\frac{\tau_{i+1 / 2}-\tau_{i-1 / 2}}{\Delta z_{i}}+R_{X_{i}} \quad i=2,3, \ldots, \mathrm{NZ}-1 .
$$

The levels $i=1$ and $i=$ NZ refer to the surface and top, respectively. For $i \geq 2, \tau_{i+1 / 2}$ is the vertical turbulent flux of $X$, parameterized using $K$ theory,

$$
\tau_{i+1 / 2}=\overline{X^{\prime} w_{i+1 / 2}^{\prime}}=K_{i+1 / 2} \frac{X_{i+1}-X_{i}}{\Delta z_{i+1 / 2}} .
$$

The parameterization of $\tau_{1+1 / 2}$, the surface flux (surface stress for momentum), is to be given special treatment, as presented in the next subsection.

To prevent numerical instability, an implicit temporal discretization is adopted for the turbulent flux terms. The details are omitted here, but with this approach the discretized system of $(\mathrm{NZ}-2)$ equations is solved by triangularizing a matrix, with boundary conditions provided at $i=1$ and $i=\mathrm{NZ}$. Nevertheless, it must be emphasized that the implicit discretization ensures numerical stability, but not accuracy, which can be effectively achieved with smaller time steps.

\section{b. The surface fluxes}

The surface interactions are computed according to the similarity theory of Monin and Obukhov (1954). This theory postulates the existence of universal functions within the surface layer for the nondimensional vertical gradients of wind, temperature, and water vapor. They are given by

$$
\begin{aligned}
& \frac{\kappa z}{u_{*}} \frac{\partial u}{\partial z}=\phi_{M}\left(\frac{z}{L}\right), \\
& \frac{\kappa z}{\theta *} \frac{\partial \theta}{\partial z}=\phi_{H}\left(\frac{z}{L}\right), \quad \text { and } \\
& \frac{\kappa z}{*} \frac{\partial q}{\partial z}=\phi_{Q}\left(\frac{z}{L}\right),
\end{aligned}
$$

where $L$ is the Monin-Obukhov length, $\kappa=0.35$ is the von Kármán constant, $u *$ is the friction velocity, and $\theta *$ and $q_{*}$ are temperature and vapor flux scales. These parameters are defined from the surface fluxes of momentum, heat, and water vapor by

$$
\begin{aligned}
& F_{M}=\rho u_{*}^{2}, \\
& F_{H}=-c_{p} \rho u_{*} \theta_{*}, \quad \text { and } \\
& F_{Q}=-\rho u_{*} q_{*},
\end{aligned}
$$

where $c_{p}=1004 \mathrm{~J} \mathrm{~K}^{-1} \mathrm{~kg}^{-1}$ is the specific heat of air at constant pressure and $\rho$ is the density of air. Equation (2.4) integrated over $z$ for the generic variable $X$ results in

$$
X(z)=X\left(z_{0}\right)+\frac{x_{*}}{\kappa}\left[\ln \frac{z}{z_{0 X}}-\psi_{X}\left(\frac{z}{L}\right)\right],
$$

where

$$
\psi_{X}\left(\frac{z}{L}\right)=\int_{z_{0 X} / L}^{z / L} \frac{1-\phi_{X}}{z^{\prime} / L} d\left(\frac{z^{\prime}}{L}\right)
$$

and the roughness length $z_{0}$ for momentum is the height $z$ where $u=0$. Here $\psi_{X}$ is used in the form presented by Pielke (2002), based on the empirical formulation of Businger et al. (1971). Andreas (2009) emphasized that some care is necessary in choosing $\kappa$ because it must be compatible with the empirical coefficients of the universal profile functions of similarity theory, which are adjusted to data from field experiments. In our case, we adopted the functions given by Businger et al. where $\kappa=0.35$.

The scalar roughness lengths $z_{0 q}$ and $z_{0 \theta}$ are given by Zeng et al. (1998) as

$$
z_{0 q}=z_{0 \theta}=\exp \left(2.57-2.67 R_{r o}^{0.25}\right)
$$

with the roughness Reynolds number $R_{r o}=u * z_{0} / \nu$, where $\nu$ is the kinematic viscosity of air. These formulations are based on the well-known Liu-Katsaros-Businger (LKB) renewal model proposed by Liu et al. (1979).

The numerical solution consists of finding $x *$ (actually $u_{*}, \theta_{*}$, and $\left.q_{*}\right)$ using Eq. (2.6), which accommodates $X$ (whose values are already known at two levels) and $z_{0}$ (modified by the wave activity and spume droplet load). Afterward, the surface fluxes are calculated with Eq. (2.5) and used in Eq. (2.2) written for the level $i=2$ to compute the prognostic variable. As $L$ and many formulations for $z_{0}$ depend on $u *$ (as will be presented), the computation of $x *$ involves iterative procedures, and simplifications for $\phi_{M}, \phi_{H}$, and $\phi_{Q}$ are required. The detailed numerical procedure is omitted here, but it is similar to the approaches described by Pielke (2002) and Krishnamurti et al. (1983). 


\section{c. The wave-induced stress and droplet load}

The inclusion of wave-induced stress and droplet load in our model is based on the approach suggested by Janssen (1991). It is assumed that near the surface the total stress, independent of $z$, defines the friction velocity $u *$ by

$$
\tau_{\text {tot }}=\tau_{\text {turb }}+\tau_{\text {wave }}+\tau_{\text {spray }}=\text { const }=\rho u_{*}^{2},
$$

where $\tau_{\text {turb }}, \tau_{\text {wave }}$, and $\tau_{\text {spray }}$ are the stresses due to the turbulent atmospheric motion, the organized air motion induced by surface waves, and the spray load. The viscous stress is implicitly incorporated by assuming the validity of Eq. (2.7) for $z$ above the viscous roughness length $z_{0}$, where $u\left(z_{0}\right)=0$. Janssen (1991) assumes $\tau_{\text {turb }} \propto$ $|\partial u / \partial z|$; so as to be consistent with the formulation for the boundary layer employed here, we assume that, in the presence of these stresses, the velocity profile given by Eq. (2.6) is modified to

$$
u(z)=\frac{u *}{\kappa}\left[\ln \left(\frac{z+z_{1}}{z_{0}+z_{1}}\right)-\psi_{M}\right],
$$

where $z_{1}$ is the contribution from the wave and spray stresses to the effective roughness length $\left(z_{0}+z_{1}\right)$. Unlike in the profile defined by Eq. (2.6), $\tau_{\text {turb }}$ depends on $z$ and is not equal to $\rho u_{*}^{2}$. The wave-induced and spray load stresses also depend on $z$, but they can be neglected for $z \geq 10 \mathrm{~m}$ (Makin et al. 1995). The profile assumed does not alter the roughness length where $u(z)=0$, and for $\gg z_{1}$ it is nearly that given by (2.6). A relation for $\tau_{\text {turb }}$ can be obtained differencing (2.8) with respect to $z$,

$$
\frac{\kappa\left(z+z_{1}\right)}{u *} \frac{\partial u}{\partial z}=\phi_{M}
$$

from which it follows that, after defining $u_{* \text { turb }} \equiv$ $(\kappa z \partial u / \partial z) / \phi_{M}$,

$$
\begin{aligned}
\tau_{\text {turb }}(z) & \equiv \rho\left(\frac{\kappa z \partial u / \partial z}{\phi_{M}}\right)^{2}=\rho\left(\frac{z}{z+z_{1}}\right)^{2}\left[\frac{\kappa\left(z+z_{1}\right) \partial u / \partial z}{\phi_{M}}\right]^{2} \\
& =\tau_{\text {tot }}\left(\frac{z}{z+z_{1}}\right)^{2}
\end{aligned}
$$

These relations show that, provided $z_{1} \geq 0$, its role is to reduce $\partial u / \partial z$ and $\tau_{\text {turb }}(z) \leq \tau_{\text {tot. }}$ Using (2.9) in Eq. (2.7), we have

$$
1=\frac{\tau_{\text {turb }}}{\tau_{\text {tot }}}+\frac{\tau_{\text {wave }}+\tau_{\text {spray }}}{\tau_{\text {tot }}}=\left(\frac{z}{z_{0}+z_{1}}\right)^{2}+\frac{\tau_{\text {wave }}+\tau_{\text {spray }}}{\tau_{\text {tot }}},
$$

which, after replacing $z$ by $z_{0}$, yields

$$
z_{1}=z_{0}\left[\sqrt{\frac{1}{1-\left(\tau_{\text {wave }}+\tau_{\text {spray }}\right) / \tau_{\text {tot }}}}-1\right] \text {. }
$$

Then, in this approach $z_{0}$ is due to viscosity evolving with the turbulent stress at the surface, whereas $z_{1}$ evolves with the wave and spray stress. Defining the turbulent friction velocity by $u *$ turb $=\left(\tau_{\text {turb }} / \rho\right)^{0.5}$, the numerical procedure is

(i) as a first guess, assume $z_{0}=0.0001 \mathrm{~m}, z_{1}=0$ and $\psi_{M}=0$ in (2.8) to compute $u *$ using $u$ at the first level above the surface $(i=2)$;

(ii) from the wave and spray parameterizations, compute $\tau_{\text {wave }}$ and $\tau_{\text {spray }}$;

(iii) from (2.10), compute $z_{1}$;

(iv) using $u$ at $i=2$ and $\psi_{M}$, compute $u *$ with (2.8);

(v) from (2.9), compute $\tau_{\text {turb }}$ and $u *$ turb at $z_{0}$;

(vi) from the surface flux parameterization adopted, use $u *$ turb to compute $z_{0}$; and

(vii) check the convergence by comparing two consecutive computations of $u *$ turb if necessary, return to the third step.

Section 3 presents the spray parameterization, and section 4 the wave and surface flux turbulent parameterizations.

\section{d. Latent and sensible heat}

This section presents the procedure for computing the mass of water $F_{\text {ejec }}$ ejected into the atmosphere, the mass $F_{\text {back }}$ that falls back into the sea [the reentrant mass, as defined by Andreas and Emanuel (2001)], and the potential net mass $F_{\text {spray }}^{\text {max }}=F_{\text {ejec }}-F_{\text {back }}$ that can be evaporated. Here we show how these quantities, given per unit of sea surface area at each second, are incorporated into the prognostic equations used by the numerical model proposed.

The exchanges of heat and moisture will be assumed to take place between the surface and the first level above, in our model defined as $10 \mathrm{~m}$. These effects are incorporated in two phases: first, an equilibrium temperature owing to sensible heat is found and, second, the temperature and mixing ratio are modified owing to the spray evaporation. This approach is overestimating the effect of sensible heat because all ejected mass is assumed to exchange sensible heat with the atmosphere before falling back to the sea; however, this assumption is reasonable because the sensible heat contribution is small, much smaller than the latent heat, as will be shown in the numerical simulations.

Since during one time step $\Delta t$ the total water mass ejected in an area $A$ is given by 


$$
M_{\text {ejec }}=F_{\text {ejec }} A \Delta t,
$$

an air column with thickness $\Delta z$ and initial temperature $T_{i}$ receiving part of this amount $P \times M_{\text {ejec }}$ with temperature $T_{\text {spray }}$ must come to a final temperature $T_{f}$, satisfying

$$
\left(T_{f}-T_{i}\right) c_{p} \rho A \Delta z=\left(T_{\text {spray }}-T_{f}\right) c_{p_{\text {water }}} A \Delta t P F_{\text {ejec }}
$$

or

$$
T_{f}=\frac{T_{i} c_{p} \Delta z \rho+P F_{\text {ejec }} T_{\text {spray }} c_{p_{\text {water }}} \Delta t}{c_{p} \Delta z \rho+P F_{\text {ejec }} c_{p_{\text {water }}} \Delta t},
$$

where $0 \leq P \leq 1$ and $c_{p_{\text {water }}}=4184 \mathrm{~J} \mathrm{~K}^{-1} \mathrm{~kg}^{-1}$ is the specific heat of water at constant pressure. Determining the vertical distribution of temperature between the surface and the first level above is a difficult matter. Although Fairall et al. (1994) assumes a standard vertical gradient of temperature, we have adopted a different approach; because the layer affected by the spray is defined by only two levels, $z=0$ and $z=10 \mathrm{~m}$, we divide it into two sublayers of equal thickness $\Delta z=5 \mathrm{~m}$, distributing $P M_{\text {ejec }}$ into the sublayer near the surface and $(1-P) M_{\text {ejec }}$ into the other; $P$ is expected to be $H_{s}$ dependent so that the influence of the ejected spray on the sublayer adjacent to the surface increases with $H_{s}$ decreasing.

As a qualitative attempt, we define a partition of $P$ for the lower sublayer as

$$
P\left(H_{s}\right)=\left\{\begin{array}{cll}
1 & \text { for } & H_{s} \leq 1 \mathrm{~m} \\
\frac{9-H_{s}}{8} & \text { for } & 1<H_{s}<5 \mathrm{~m} \\
0.5 & \text { for } & H_{s} \geq 5 \mathrm{~m}
\end{array}\right.
$$

where it can be seen that for $H_{s} \leq 1 \mathrm{~m}$ no increment of temperature is applied to the level $z=10 \mathrm{~m}$ and for $H_{s} \geq 5 \mathrm{~m}$ the same increment is applied to both levels.

Concerning the spray evaporated, it cannot be greater than the maximum available nor can it yield a supersaturated layer. If there is no efficient physical mechanism to remove vapor, the evaporation will continue while the waves are breaking and the atmosphere is not saturated. Although the computation of mass evaporated takes account of the atmospheric relative humidity, approximations in the numerical procedures can result in a supersaturated layer. To prevent this problem, the supersaturation is removed after submitting the saturated layer to a pseudoadiabatic process as described below. A similar procedure was adopted by Innocentini and Neto (1992).

As the curvature effect on the saturation pressure is nearly null for radius greater than $10 \mu \mathrm{m}$ (Pruppacher and Klett 1978, Fig. 6.2), the saturation mixing ratio over a plane water surface based on the Teten formula (Soong and Ogura 1973) is evoked,

$$
q_{\mathrm{sat}}(T, p)=\frac{3.8}{p} \exp \left[\frac{a \ln 10 \times(T-273)}{T-36}\right],
$$

where $a=7.5$ and the pressure $p$ is in millibars. For a small temperature increment $\Delta T$, the expansion of $q_{\text {sat }}$ in a Taylor series, retaining only the first-order term, results in

$$
q_{\mathrm{sat}}(T+\Delta T)=q_{\mathrm{sat}}(T)+\left.\Delta T \frac{\partial q_{\mathrm{sat}}}{\partial T}\right|_{T}
$$

with

$$
\left.\frac{\partial q_{\mathrm{sat}}}{\partial T}\right|_{T}=q_{\mathrm{sat}}(T)\left[\frac{a \ln 10 \times 237}{(T-36)^{2}}\right] .
$$

On the other hand, a parcel initially with mixing ratio $q$ and temperature $T$, which is saturated by a pseudoadiabatic process that changes its temperature to $(T+$ $\Delta T)$, must satisfy the equation

$\left[q_{\text {sat }}(T+\Delta T)-q(T)\right] L_{\text {water }}=-c_{p_{\text {water }}} \Delta T$,

where $L_{\text {water }}=2.5 \times 10^{6} \mathrm{~J} \mathrm{~kg}^{-1}$ is the latent heat of water vaporization. This equation combined with Eq. (2.14) yields

$$
q_{\mathrm{sat}}(T+\Delta T)=q(T)+\frac{q_{\mathrm{sat}}(T)-q(T)}{1+\Psi},
$$

where

$$
\Psi \equiv \frac{a \ln 10 \times 237 q_{\text {sat }}(T) L_{\text {water }}}{c_{p_{\text {water }}}(T-36)^{2}} .
$$

The amount of vapor necessary to saturate a column with volume $A \Delta z$ is

$$
E_{\text {spray }}^{\max }=\left[q_{\text {sat }}(T+\Delta T)-q(T)\right] \rho A \Delta z,
$$

which is the maximum water allowed to be evaporated into the column. Then, the total mass evaporated into a column is the smaller of $E_{\text {spray }}^{\max }$ or the net mass (ejected minus returned) by the breaking waves. Following the same approach employed in the partition of sensible heat, in one time step $\Delta t$ the mass evaporated into each sublayer is given by

$$
M_{\text {evap }}=\min \left[\delta \times\left(M_{\text {ejec }}-M_{\text {back }}\right), \Delta t \times E_{\text {spray }}^{\max }\right],
$$

where $\delta$ is $P$ for the near-surface sublayer and $(1-P)$ for the upper layer; $E_{\text {spray }}^{\max }$ refers to the appropriate sublayer. 
Then the mixing ratio and temperature at each level is given by

$$
\begin{aligned}
& q_{f}(z)=\frac{M_{\text {evap }}}{\rho A \Delta z}+q_{i}(z) \\
& T_{f}(z)=\left[q_{f}(z)-q_{i}(z)\right] \frac{L_{\text {water }}}{c_{p_{\text {water }}}}+T_{i}(z),
\end{aligned}
$$

with $A=1 \mathrm{~m}^{2}$ and $\Delta z=5 \mathrm{~m}$.

\section{Approximate formulas for spume droplets}

The objective of this research is also to develop a numerical parameterization for the air-sea exchange processes to be implemented in operational models. For this, it seems better to avoid computing the evolution of each droplet at each time step. This section presents an efficient approach in which the collective role of spume droplets is stored as a function of air temperature, relative humidity, and significant wave height; the effective latent and sensible heat contribution is given by recovering this value and multiplying it by the windsea Reynolds number.

\section{a. The spume droplet spectrum}

If $F(r)$ represents the number flux of droplets with radius $r$ ejected into the atmosphere, the SSGF is defined by $d F / d r$ and the integral

$$
\int_{r_{L}}^{r_{H}} \frac{d F}{d r} d r
$$

yields the number flux of droplets with radius between $r_{L}$ and $r_{H}$, corresponding to the mass flux

$$
F_{\text {spray }}=\frac{4 \pi}{3} \rho_{\text {spray }} \int_{r_{L}}^{r_{H}} r^{3} \frac{d F}{d r} d r .
$$

Toba et al. (2006) assume SSGF as a product of two functions, $f_{1}$ depending on the wind speed and wind waves and $f_{2}$ modeling the shape of the droplet spectrum:

$$
\frac{d F}{d r}=f_{1}\left(U_{10}, \sigma_{p}\right) f_{2}(r)
$$

$U_{10}$ being the wind speed at the $10-\mathrm{m}$ height and $\sigma_{p}$ the peak angular frequency of the windsea wave spectrum. The function $f_{1}\left(U_{10}, \sigma_{p}\right)$ has been simplified by many authors to $f_{1}\left(U_{10}\right)$ (e.g., Wu 1973; Ling et al. 1980). However, Toba et al. pointed out that the windsea Reynolds number $R_{B}$, defined by

$$
R_{B}=\frac{u_{*}^{2}}{\sigma_{p} \nu}
$$

is the most appropriate controlling parameter for airsea interaction and droplet production; $\nu=1.326 \times$ $10^{-5} \mathrm{~m}^{2} \mathrm{~s}^{-1}$ is the kinematic viscosity of air.

Zhao et al. (2006) assumed $R_{B}>10^{3}$ as the threshold for spume production and expressed $f_{1}$ in the form

$$
f_{1}\left(R_{B}\right)=a R_{B}^{b}
$$

with $a=8.39 \times 10^{-3}$ and $b=1.50$ fitting better the data provided by several observational campaigns covering droplet radius and wind $U_{10}$ ranging up to $873 \mu \mathrm{m}$ and $41.3 \mathrm{~m} \mathrm{~s}^{-1}$.

The spectral shape proposed by Monahan et al. (1986) has been accepted by many researchers (e.g., Andreas 1998; Wu 1998). From it, the SSGF assumes the relation

$$
\frac{d F}{d r}=f_{1}\left(R_{B}\right) f_{2}(r)=R_{B}^{1.5} \times\left\{\begin{array}{cc}
a_{1} r^{-1} & 30<r<75 \mu \mathrm{m} \\
a_{2} r^{-3} & 75<r<200 \mu \mathrm{m} \\
a_{3} r^{-8} & 200<r<500 \mu \mathrm{m}
\end{array}\right.
$$

expressed in $\mathrm{m}^{-2} \mathrm{~s}^{-1} \mu \mathrm{m}^{-1}$, where

$$
\begin{aligned}
& a_{1}=7.84 \times 10^{-3}, \\
& a_{2}=4.41 \times 10,
\end{aligned}
$$

and

$$
a_{3}=1.41 \times 10^{13} .
$$

Therefore, Eq. (3.2) can be analytically integrated with $d F / d r$ above, so the numerical evaluation of $F_{\text {spray }}$ depends only on the wave spectrum and wind stress (represented by $\sigma_{p}$ and $u *$, respectively); the result for the integral in this equation, from $r_{L}=30 \mu \mathrm{m}$ to $r_{H}=$ $500 \mu \mathrm{m}$, is $8.691 \times 10^{-15} R_{B}^{1.5} \mathrm{~m} \mathrm{~s}^{-1}$.

\section{b. Spray stress}

Andreas (2004) assumed that the droplets reach the airspeed in less than $1 \mathrm{~s}$, meaning that all launched droplets extract momentum and slow the wind before returning to the sea. The stress induced is

$$
\tau_{\text {spray }}=\frac{4 \pi}{3} \rho_{\text {spray }} \int_{r_{L}}^{r_{H}} u_{\text {spray }}(r) r^{3} \frac{d F}{d r} d r
$$

with the droplet horizontal velocity $u_{\text {spray }}$ computed at the effective height $z_{\text {spray }}$ where the spume droplet of radius $r$ is produced. The radii $r_{L}=30 \mu \mathrm{m}$ and $r_{H}=500 \mu \mathrm{m}$ are the limits assumed for spume size spectrum. Andreas (1992) and Iida et al. (1992) suggested adopting a $z_{\text {spray }}$ representative for all droplets, as the fraction of the significant wave height; we are using Iida et al.'s value 


$$
z_{\text {spray }}=0.63 H_{s}
$$

The value of $H_{s}$ is computed from the wave spectrum and $u_{\text {spray }}$ is estimated from the logarithmic profile

$$
u_{\text {spray }}=\frac{u_{*}}{\kappa} \ln \left(\frac{z_{\text {spray }}}{z_{0}}\right),
$$

with the roughness length $z_{0}$ and the friction velocity $u *$ given by the atmospheric boundary layer model. Therefore, Eq. (3.4) can be written as

$$
\tau_{\text {spray }}=\frac{4 \pi}{3} \rho_{\text {spray }} u_{\text {spray }} \int_{r_{L}}^{r_{H}} r^{3} \frac{d F}{d r} d r
$$

for which the result for the integral was presented above. In summary, from a wind profile and a wave spectrum, $u_{\text {spray }}$ is obtained with (3.5) and $\tau_{\text {spray }}$ with (3.6).

\section{c. The time evolution of a droplet}

Following Andreas (2005), the time evolution of a droplet with initial radius $r_{i}$ is assumed to be of the form

$$
\frac{r(t)-r_{\mathrm{eq}}}{r_{i}-r_{\mathrm{eq}}}=\exp \left(\frac{-t}{\tau_{e}}\right),
$$

where $\tau_{e}$, a kind of $e$-folding time, is the time necessary to reduce the ratio of the lhs of this equation to $1 / e$, and $r_{\text {eq }}$ is the equilibrium radius achieved when the droplet is in equilibrium with the wet air. Andreas (2005) evoked Pruppacher and Klett's (1978) Eqs. (13)-(28) to describe the time rate of change of droplet radius:

$$
\frac{\partial r}{\partial t}=\frac{\zeta}{r \eta}
$$

where

$$
\begin{aligned}
\zeta & \equiv\left(R_{F}-1\right)-Y ; \\
\eta & \equiv \frac{\rho_{\text {spray }} R T}{D_{\text {water }}^{\prime} M_{\mathrm{H}_{2} \mathrm{O}} e_{\text {sat }}(T)}+\frac{\rho_{\text {spray }} L_{\text {water }}}{K_{\text {air }}^{\prime} T}\left(\frac{L_{\text {water }} M_{\mathrm{H}_{2} \mathrm{O}}}{R T}-1\right) ; \\
Y & \equiv \frac{2 M_{\mathrm{H}_{2} \mathrm{O}} \sigma_{\text {sea }}}{R T \rho_{\text {water }} r}-\frac{\nu_{\text {ion }} \Phi_{\text {spray }} m_{\text {salt }}\left(M_{\mathrm{H}_{2} \mathrm{O}} / M_{\mathrm{NaCl}}\right)}{\left(4 \pi \rho_{\text {spray }} r^{3} / 3\right)-m_{\text {salt }}} ;
\end{aligned}
$$

and $R_{F}$ is the fractional relative humidity (i.e., $0 \leq$ $\left.R_{F} \leq 1\right) ; R=8.31 \mathrm{~J} \mathrm{~mol}^{-1} \mathrm{~K}^{-1}$ the universal gas constant; $M_{\mathrm{H}_{2} \mathrm{O}}=18.016 \times 10^{-3} \mathrm{~kg} \mathrm{~mol}^{-1}$ the water molecular weight; $M_{\mathrm{NaCl}}=58.443 \times 10^{-3} \mathrm{~kg} \mathrm{~mol}^{-1}$ the molecular weight of sodium chloride; $D_{\text {water }}^{\prime}$ the molecular diffusivity for water vapor; $e_{\text {sat }}$ the saturation vapor pressure for water; $K_{\text {air }}^{\prime}$ the thermal conductivity of air; $\sigma_{\text {sea }}$ is the surface tension of a flat water surface; $\Phi_{\text {spray }}$ is the osmotic coefficient; $\nu_{\text {ion }}=2$ the number of ions into which a molecule of sodium chloride dissociates; and $m_{\text {salt }}$ the salt mass in the droplet. The formulations for $K_{\text {air }}^{\prime}$ $D_{\text {water }}^{\prime} m_{\text {salt }}, \Phi_{\text {spray }}$, and $\sigma_{\text {sea }}$ adopted here are the same as those presented by Andreas (2005), based on Pruppacher and Klett (1978). For $e_{\text {sat }}$, we are using the Teten relation (Soong and Ogura 1973),

$$
e_{\mathrm{sat}}(T)=611 \exp \left(\frac{17.27 T}{T+237.3}\right)
$$

where the temperature is given in Celsius and the water pressure in pascals. The equilibrium radius $r_{\mathrm{eq}}$ corresponds to $r$ satisfying $\partial r / \partial t=0$ or $\zeta(r)=0$. Although $R_{F}$ changes while the radius is decreasing, this will be neglected in evaluating $r_{\text {eq }}$, as in Andreas (2005). Since $Y$ depends on $r$ in the denominator of both terms, an interactive method is required to solve the equation $\zeta\left(r_{\text {eq }}\right)=0$. As in Andreas (2005), the Newton iterative method can be evoked. Supposing $r_{\text {ig }}$ is the initial guess, the method yields

$$
\begin{aligned}
r_{\mathrm{eq}}^{0} & =r_{\mathrm{ig}} \\
r_{\mathrm{eq}}^{1} & =r_{\mathrm{eq}}^{0}-\frac{\zeta\left(r_{\mathrm{eq}}^{0}\right)}{\left.(\partial \zeta / \partial r)\right|_{\mathrm{r}_{\mathrm{eq}}^{0}}} \\
\vdots & \\
r_{\mathrm{eq}}^{n} & =r_{\mathrm{eq}}^{n-1}-\frac{\zeta\left(r_{\mathrm{eq}}^{n-1}\right)}{\left.(\partial \zeta / \partial r)\right|_{r_{\mathrm{eq}}-1}},
\end{aligned}
$$

where

$$
\begin{aligned}
\frac{\partial \zeta}{\partial r}= & \frac{2 M_{\mathrm{H}_{2} \mathrm{O}} \sigma_{\text {sea }}}{R T \rho_{\text {water }} r^{2}} \\
& -\frac{\nu_{\text {ion }} \Phi_{\text {spray }} m_{\text {salt }}\left(M_{\mathrm{H}_{2} \mathrm{O}} / M_{\text {NaCl }}\right)\left(4 \pi r^{2} \rho_{\text {spray }}\right)}{\left[4 \pi r^{3} \rho_{\text {spray }} / 3-m_{\text {salt }}\right]^{2}} .
\end{aligned}
$$

One can note a discontinuity in $\partial \zeta / \partial r$ when the denominator of the second term is zero. This means that the initial guess of $r$ is crucial if the iterative method is to converge to the correct value. The divergence will occur if the first guess is smaller than the $r$ corresponding to the discontinuity. To avoid this problem the initial guess is $(r+0.1 r)$, with

$$
r=\left(\frac{3 m_{\text {salt }}}{4 \pi \rho_{\text {spray }}}\right)^{1 / 3}
$$

The $e$-folding time $\tau_{e}$ can be evaluated from the following procedure. For a function $H(t)$ defined by 


$$
H(t)=\ln \left(\frac{r(t)-r_{\mathrm{eq}}}{r_{i}-r_{\mathrm{eq}}}\right),
$$

one can observe that $H\left(\tau_{e}\right)=-1$ and $H(0)=0$. Expanding $H(t)$ in Taylor series around $t=\tau_{e} / 2$,

$$
\begin{gathered}
H(0)=H\left(\tau_{e} / 2\right)-\left.\frac{\tau_{e}}{2} \frac{\partial H}{\partial t}\right|_{\tau_{e} / 2}+\left.\left(\frac{\tau_{e}}{2}\right)^{2} \frac{\partial^{2} H}{\partial t^{2}}\right|_{\tau_{e} / 2}-\cdots ; \\
H\left(\tau_{e}\right)=H\left(\tau_{e} / 2\right)+\left.\frac{\tau_{e}}{2} \frac{\partial H}{\partial t}\right|_{\tau_{e} / 2}+\left.\left(\frac{\tau_{e}}{2}\right)^{2} \frac{\partial^{2} H}{\partial t^{2}}\right|_{\tau_{e} / 2}+\cdots
\end{gathered}
$$

and subtracting these two expressions, neglecting terms of order 3 and higher, the result is

$$
H\left(\tau_{e}\right)-H(0)=\tau_{e} \times\left.\frac{\partial H}{\partial t}\right|_{\tau_{e} / 2}=-1
$$

or

$$
\tau_{e}=-\left(\left.\frac{\partial H}{\partial t}\right|_{\tau_{e} / 2}\right)^{-1}
$$

From the definition of $H(t)$ we have

$$
\left.\frac{\partial H}{\partial t}\right|_{\tau_{e} / 2}=\left(\frac{r_{i}-r_{\mathrm{eq}}}{r\left(\tau_{e} / 2\right)-r_{\mathrm{eq}}}\right) \times\left.\frac{\partial r}{\partial t}\right|_{\tau_{e} / 2}
$$

with $\partial r / \partial t$ given by (3.8).

To estimate $\left.(\partial H / \partial t)\right|_{\tau_{e} / 2}$ it is necessary to know $r\left(\tau_{e} / 2\right)$ and $\left.(\partial r / \partial t)\right|_{\tau_{e} / 2}$. Since $H(t)$ is nearly linear from $H(0)=0$ to $H\left(\tau_{e}\right)=-1$, it is reasonable to assume that $H\left(\tau_{e} / 2\right)=$ $-1 / 2$; thus, we have

$$
\exp \left[H\left(\tau_{e} / 2\right)\right]=\frac{r\left(\tau_{e} / 2\right)-r_{\mathrm{eq}}}{r_{i}-r_{\mathrm{eq}}}=\sqrt{\frac{1}{e}}
$$

or

$$
r\left(\tau_{e} / 2\right)=\sqrt{\frac{1}{e}} \times\left(r_{i}-r_{\mathrm{eq}}\right)+r_{\mathrm{eq}} .
$$

For each radius the numerical procedure consists in applying the iterative method (3.9) for computing $r_{\text {eq }}$. Thus, $r\left(\tau_{e} / 2\right)$, given by Eq. (3.12), allows the evaluation of $(\partial r / \partial t) \mid \tau_{e} / 2$. using Eq. (3.8), and finally $\tau_{e}$ is obtained with Eq. (3.10).

\section{d. Sea spray evaporated mass}

If the evaporation process reduces a droplet from its initial radius $r_{i}$ to $r_{f}$, the total mass reduction per unit area of sea surface per second is

$$
F_{\text {evap }}=\rho_{\text {spray }} \int_{r_{L}}^{r_{H}}\left(r^{3}-r_{f}^{3}\right)\left(\frac{4 \pi}{3} \frac{d F}{d r}\right) d r .
$$

The value of $r_{f}$ is given by (3.7), which depends on the residence time of each droplet: if the time necessary to reduce $r_{i}$ to $r_{\mathrm{eq}}$ is less than it, then $r_{f}=r_{\mathrm{eq}}$; otherwise $r_{f}>$ $r_{\text {eq. }}$. The residence time depends on the droplet radius and height where it is released into the atmosphere. Andreas (1992) considered the gravitational force and air drag on a falling droplet with the drag coefficient proposed by Friedlander (1977, p. 105); the final droplet speed thus obtained is

$$
U_{f}=\frac{2 r^{2} g}{9 \nu\left[1+0.158\left(2 r U_{f} / \nu\right)^{2 / 3}\right]}\left(\frac{\rho_{\mathrm{water}}}{\rho}-1\right),
$$

where $R_{e}=2 r U / \nu$ is the traditional Reynolds number. This transcendental equation is solved for $U_{f}$ applying the Newton iterative method. Then, for a droplet launched into the atmosphere at $z=Z$, the residence time is $Z / U_{f}$.

Taking the droplet spectrum given by Eq. (3.3), we have

$$
F_{\text {evap }}=\frac{4 \pi}{3} \rho_{\text {spray }} R_{B}^{1.5}\left(\phi_{1}-\phi_{2}\right)
$$

in which

$$
\begin{aligned}
& \phi_{1}=\int_{r_{L}}^{r_{H}} r^{3} f_{2}(r) d r ; \\
& \phi_{2}=\int_{r_{L}}^{r_{H}} r_{f}^{3} f_{2}(r) d r .
\end{aligned}
$$

The value of the first integral was presented at the end of section $3 \mathrm{a}$, while the second can be calculated by its discretized formulation.

\section{e. Numerical integration procedures}

The treatment of the effects of the droplets in the numerical model relies on both the amount of mass ejected into the atmosphere (so as to evaluate the sensible heat and spray stress) and the mass evaporated, which depends on the evolution of the spectrum (so as to evaluate the latent heat released).

The estimation of the mass ejected does not offer any difficulty, because $F_{\text {spray }}$ is given by Eq. (3.2), with $8.691 \times 10^{-15} R_{B}^{1.5} \mathrm{~m} \mathrm{~s}^{-1}$ replacing the integral and $R_{B}^{1.5}$ evaluated from the wave and atmospheric models at each time step. On the other hand, the mass evaporated $\phi_{2}$ in Eq. (3.13) requires more complex computations. Instead of computing $\phi_{2}$ each time step, we decided to prepare a table so its value can be recovered as a function 
of $T, R_{F}$, and $H_{s}$. To prepare the table, first it is necessary to compute $r_{\text {eq }}$ for each $r_{i}$ from 30 to $500 \mu \mathrm{m}$, which depends on $R_{F}, T$, and salinity $S$. However, we assume $S=$ $0.034=$ const, and $r_{\text {eq }}$ is computed from $T=273$ to $300 \mathrm{~K}$, each $1 \mathrm{~K}$, and $R_{F}$ from 0.1 to 1 , each 0.01 . The bulk molecular diffusivity of water vapor in air $D_{w}$ is computed with $T_{0}=273.15 \mathrm{~K}$, and $p_{0} / p=1.0135$. The value of the surface tension $\sigma_{\text {water }}\left(0.00751 \mathrm{~J} \mathrm{~m}^{-2}\right)$ for pure water at $T=273.15 \mathrm{~K}$ is used.

After expressing $r_{\text {eq }}$ as a function of $T$ and $R_{F}$, the next step is to compute the $e$-folding time $\tau_{e}$, so the evolution of each droplet radius can be described by Eq. (3.7). For this, it is required to evaluate $r\left(\tau_{e} / 2\right)$ using Eq. (3.12), $\partial \zeta /\left.\partial r\right|_{\tau_{/ 2}}$ from Eq. (3.8), and $\partial H /\left.\partial t\right|_{\tau_{/ 2}}$ from Eq. (3.11). Finally, $\tau_{e}$ given by Eq. (3.10) can be estimated for the same range of $T$ and $R_{F}$ used above.

The final requirement for computing $\phi_{2}$ is to know $r_{f}$, which depends not only on $T$ and $R_{F}$ but also on the residence time. We compute the residence time, as presented above, for $H_{s}=1,2,3,4,5$, and $6 \mathrm{~m}$, and finally $\phi_{2}$ can be stored in the table for the same range of $T, R_{F}$, and $H_{S}$ proposed above. We noted that, for $T$ and $R_{F}$ constants, the variation of $\phi_{2}$ is nearly linear with $H_{s}$. Then, during the model integration, a linear interpolation is used to compute $\phi_{2}$ at intermediate values of $H_{s}, T$, and $R_{F}$.

\section{Wave stress}

The wave stress implies momentum transfer from the atmosphere due to the decrease of surface wind velocity caused by the surface waves. In section $2 c$, we emphasized the distinction between friction velocity $u_{*}$ defined from total stress and from turbulent stress; in this section, we write $u_{*}$ referring to the turbulent stress. Charnock (1955) suggested the parameterization of the sea surface effect on the atmosphere with the surface roughness $z_{0}$ in the form

$$
z_{0}=\alpha \frac{u_{*}^{2}}{g}, \quad \alpha=0.0185
$$

Later, analysis of laboratory and field observations revealed that $\alpha$ could not be considered constant. Several relations have been proposed, some using properties of the wave spectrum (e.g., wave age and wave steepness) and others employing directly the energy transferred by the wind to the windsea spectrum. In this aspect, much research has shown that most of the wave surface stress is due to the high frequency spectral components (e.g., Makin and Kudryavtsev 1999; Donelan 1998); this can be simulated by a numerical model only if it considers these components explicitly instead of derived parameters (such as wave age or wave steepness). Then, wave age and wave steepness are regarded here as less accurate parameterizations: to know the consequences of their implementation is an important aspect.

\section{a. Wave age and wave steepness}

Smith et al. (1992), examining the data of Humidity Exchange over the Sea (HEXOS), found that

$$
\alpha=0.48\left(\frac{u *}{C_{p}}\right),
$$

where $C_{p}$ is the phase speed of the peak frequency of the wave spectrum, is a more appropriate relation. Volkov (2001) examined a greater volume of data and concluded that

$\alpha=\left\{\begin{array}{ll}0.03 \frac{C_{p}}{u *} \exp \left[-0.14\left(\frac{C_{p}}{u_{*}}\right)\right], & 0.35<\frac{C_{p}}{u_{*}}<35 \\ 0.008, & \text { otherwise }\end{array}\right.$.

Since $C_{p} / u_{*}$ increases as the wave spectrum matures, it is referred to as wave age.

In contrast with these two relations using the wave age, Taylor and Yelland (2001) found good agreement between the observations and the relation using the wave steepness given by

$$
z_{0}=H_{s} \times a\left(\frac{H_{s}}{L_{p}}\right)^{b}, \quad a=1200, \quad b=4.5,
$$

where $L_{p}$ is the wavelength at peak frequency. The ratio $H_{s} / L_{p}$ represents the steepness of the wave. In general, for old waves one should expect small steepness and high wave age, and the opposite for young waves.

\section{$b$. Wind action on the wave spectrum}

A more complex parameterization of wave-induced stress, where the total source of energy to the wave spectrum is employed to computing the stress, was used by Janssen (1989). Let $E(\theta, \sigma)$ be the wave variance density spectrum, where $\theta$ is the direction and $\sigma$ is the angular frequency. The wave momentum $W_{m}$ is (Janssen 1989)

$$
W_{m}(\theta, \sigma)=\rho_{\text {water }} \sigma E(\theta, \sigma),
$$

and its time derivative owing to the wind action defines the stress: if the wind direction is $\theta_{\text {wind }}$, the rate of $W_{m}$ owing to its action becomes

$$
\tau_{\text {wave }}=\rho_{\text {water }} \int_{0}^{2 \pi} \int_{0}^{\infty} \sigma \frac{d E}{d t} d \sigma d \theta, \quad\left|\theta_{\text {wind }}-\theta\right| \leq \pi / 2,
$$


where $\tau_{\text {wave }}$ is the wave-induced stress [Holthuijsen 2007 , p. 291, Eq. (9.3.16)]. If a wave model is being integrated, only the wind's contribution to the input of energy at the rate of $E(\theta, \sigma)$ must be considered in (4.5). In general, wave models cut the frequency domain at a value $\sigma_{\text {cut }}$; however, higher frequencies, not explicitly resolved by the wave model, have a considerable contribution to the effect of wave stress on the airflow (Janssen 1991). Thus, for convenience, the wave stress can be separated into two parts,

$$
\tau_{\text {wave }}=\tau_{w a_{L}}+\tau_{w a_{H}},
$$

where the first and second rhs terms refer to Eq. (4.5) integrated from 0 to $\sigma_{\text {cut }}$ and from $\sigma_{\text {cut }}$ to $\infty$, respectively. Because the wave spectrum is not represented by the wave model for $\sigma \geq \sigma_{\text {cut }}$, a distribution of energy must be assumed. Following Janssen (1992), it is given by

$$
E(\theta, \sigma)=\left(\frac{\sigma_{\text {cut }}}{\sigma}\right)^{5} E\left(\theta, \sigma_{\text {cut }}\right) \text { for } \sigma \geq \sigma_{\text {cut }} .
$$

In wave numerical models, the source term $S_{\text {in }}(\theta, \sigma)$ due to wind action is parameterized by the relation given by Phillips (1957) and Miles (1957):

$$
\left.\frac{d E}{d t}\right|_{\text {wind }}=S_{\text {in }}(\theta, \sigma)=\alpha_{w}+\beta_{w} E(\theta, \sigma),
$$

where $\alpha_{w}$ and $\beta_{w}$ are the coefficients leading to a linear growth and to an exponential growth of $E$, respectively. As suggested by Cavaleri and Malanotte-Rizzoli (1981), the linear term $\alpha$ has the form

$\alpha_{w}=\left\{\begin{array}{ll}\frac{\rho}{2 \pi g^{2}}(u * \cos \theta)^{4} \times \exp \left(-\frac{\sigma}{\sigma_{\text {peak }}}\right), & \left|\theta-\theta_{\text {wind }}\right| \leq 90^{\circ} \\ 0, & \text { otherwise }\end{array}\right.$,

where $\sigma_{\text {peak }}=(2 \pi \times 0.13 g) /(28 u *)$ is the peak angular frequency for the fully developed spectrum. The exponential growth term $\beta_{w}$, as given by Janssen (1992), is written

$$
\beta_{w}=\sigma \frac{\rho}{\rho_{\text {water }}}\left[\frac{1.2}{K^{2}} \epsilon \ln ^{4}(\epsilon)\right]\left(\frac{u_{*} \cos \theta}{C}\right)^{2}
$$

where

$$
\epsilon \equiv\left(\frac{u *}{\kappa C}\right)^{2}\left(\frac{g \kappa^{2}\left(z_{0}+z_{1}\right)}{u_{*}^{2}}\right) \exp \left(\frac{\kappa C}{u_{*} \cos \theta}\right),
$$

$C$ is the phase speed of the component with frequency $\sigma$, and $\left(z_{0}+z_{1}\right)$ is the effective roughness length given in section 2c. The numerical procedure computes $\tau_{w a_{L}}+$ $\tau_{w a_{H}}$ using the discretized integral in the form

$$
\tau_{\text {wave }}=\rho_{\text {water }} \sum_{\sigma} \sum_{\theta} \sigma[E(\theta, \sigma) \times \beta(\theta, \sigma)+\alpha(\theta, \sigma)] \Delta \theta \Delta \sigma,
$$

with $\sigma$ discretized from the minimum model value to a maximum value, where the increment in the integral becomes nearly zero.

\section{Numerical experiments}

The experiments consist of defining an initial profile and roughness length $z_{0}$ and representing an artificial meteorological event by the terms $R_{X}$ in Eq. (2.1) that are able to maintain the initial state in equilibrium. The initial condition is constituted by a wind speed $15 \mathrm{~m} \mathrm{~s}^{-1}$, potential temperature $280 \mathrm{~K}$, and relative humidity $90 \%$. At elevation $z_{0}$ a wind speed of zero is imposed. The sea temperature and salinity are maintained constant: $285 \mathrm{~K}$ and 0.034 . The air temperature at surface is initially $285 \mathrm{~K}$, which makes the surface layer statically unstable. A very small roughness length $z_{0}=0.0001 \mathrm{~m}$ is imposed to maintain the initial profile.

The equilibrium is broken by a windsea spectrum, and the model is integrated until a new steady state is achieved with no modification of the wave spectrum. Of course, in nature it is impossible to keep an initial profile in steady state over the sea with a constant $z_{0}$ because the equilibrium is obtained only when the wave spectrum achieves its fully developed stage determined by the local wind, interfering in $u *$ and $z_{0}$ and modifying the atmospheric profile itself. However, the maintenance of a constant wave field during the integration is a reasonable assumption when one realizes that the atmospheric response to surface interference is immediate, while a steady wave spectrum is established only after several hours or days; our numerical experiments show the atmosphere achieving a steady state in less than $1 \mathrm{~h}$.

\section{a. Wave stress}

The numerical experiments consist of breaking the initial balance by imposing a spectrum at a fully developed stage generated in a remote region: that is, not associated with the local wind speed. The incoming spectrum will be characterized by its peak period $T_{p}$, varying from 2 to $20 \mathrm{~s}$. Each $T_{p}\left(=1 / f_{p}\right)$ defines a Joint North Sea Wave Project (JONSWAP) spectrum in the form

$$
F(f)=\alpha_{e} \frac{g^{2}}{(2 \pi)^{4} f^{5}} \exp \left\{-\frac{5}{4}\left(\frac{f_{p}}{f}\right)^{4}\right\} \gamma^{\exp \left[-12\left[\left(f-f_{p}\right) /\left(\sigma f_{p}\right)\right]^{2}\right]}
$$


with $\alpha_{e}=0.0081, \sigma=0.07$ (0.09) for $f \leq f_{p}\left(f>f_{p}\right)$, and $\gamma=3.3$ (Holthuijsen 2007). In the numerical model, this spectrum is discretized in 36 directions and 25 frequencies, defined by

$$
\begin{aligned}
& \theta_{i}=i \times 10 \quad i=10, \ldots, 36, \\
& f_{i}=f_{1} \times c^{(i-1)} \quad i=1, \ldots, 25,
\end{aligned}
$$

with $f_{1}=0.03093 \mathrm{rad} \mathrm{s}^{-1}$ and $c=1.1$; these frequencies correspond to periods from 2.5 to $32.3 \mathrm{~s}$. Because the first model period is $2.5 \mathrm{~s}$, experiments with peak period equal to $2 \mathrm{~s}$ has nearly no wave energy.

We will refer to the wave steepness (Taylor and Yelland 2001), wave age (Volkov 2001), and wind input of energy (Janssen 1991) parameterizations presented in section 4 as WaSt, $\mathrm{WaAg}$, and WiAc, respectively. Before considering the model results, it is instructive to carry out some discussion about the parameterizations.

In the classical wave-age scaling $\left(C_{p} / u_{*}\right.$ or $\left.C_{p} / U_{10}\right)$, a wave spectrum is defined as young while its characteristic phase speed is lower than the wind; that is, $C_{p} / U_{10}<1$. When $C_{p} / U_{10} \approx 1$, the spectrum achieves a mature stage and the source terms (energy input, dissipation, and nonlinear transference) are in balance. When this scale is greater than 1, the spectrum is considered old. However, the wave age definition using the friction velocity $u_{*}$ rather than $U_{10}$ is more appropriate because it includes the stratification of the atmosphere and eliminates the specification of a reference height for the wind. Using this scale, a wave spectrum is mature in the range $20 \leq$ $C_{p} / u_{*} \leq 40$ (Drennan et al. 2005).

A correspondence between wave age and wave steepness scales was presented by Sverdrup and Munk (1947). They plotted observed data of steepness $H_{s} / L_{p}$ versus $C_{p} / U_{10}$; the steepness increases with $C_{p} / U_{10}$ increasing, achieves a maximum (nearly 0.1 ) for $C_{p} / U_{10} \approx 0.4$, diminishes with $C_{p} / U_{10}$ increasing up to $\approx 1.4$, and stays constant for $C_{p} / U_{10}>1.4$. Then, we can infer that the wave steepness $H_{s} / L_{p}$ scaling is high during early wave development and decreases as the spectrum matures. Carter (1982), considering the JONSWAP fully developed spectrum for deep water, estimated $H_{s}=0.0248 U_{10}^{2}$ and $T_{p}=0.729 U_{10}$, which can be used to compute $L_{p}$ from the dispersion relation $L=\mathrm{g} T^{2} /(2 \pi)$. From these relations, it follows that $H_{s} / L_{p}=0.0299$ for the fully developed stage, which can be considered the separating value between an old and young spectrum.

Laboratory and field tests with these two scalings show that both approaches have limitations in reproducing the data collected. Drennan et al. (2005), comparing these two approaches employing data from several experiments, recommended the threshold $H_{s} / L_{p}=0.02$, below which the steepness parameterization given by Eq. (4.4) produces underestimated $z_{0}$. For younger waves $\left(C_{p} / u_{*}<\right.$ $20)$, they concluded that the data are better reproduced by the wave age formulation. Then, from their research we can expect some satisfactory results using a steepness scale only for old waves or swell.

Although the two scalings discussed above employ the spectrum parameters $H_{s}, L_{p}$, and $T_{p}$, the WiAc parameterization needs the spectral description and seems to be much more sensitive to high than low frequencies, meaning that swell and old waves will have minimal impact on the wave stress, in agreement with the observations of Makin and Kudryavtsev (1999) and Donelan (1998).

The use of the deep water dispersion equation to associate the peak period and peak wavelength requires some consideration; Plant (2009) mentioned that, in general, these quantities cannot be related by this equation, which provides a wavelength smaller than the true value. The magnitude of the error depends on the spectrum shape and it is greater for wider distribution of energy among the frequencies [large $\gamma$ in Eq. (5.1) establishes a wider distribution]. Inspection of his figures shows that, for the JONSWAP spectrum with $\gamma=10$, the wavelength given by the dispersion relation is roughly $20 \%$ smaller. Then, as the wavelength in this research is obtained from the peak period through the dispersion relation, it must be regarded as an underestimate of the true value.

\section{1) TAYLOR-YELLAND PARAMETERIZATION (WAST)}

Once the steepness for the fully developed spectrum in deep water is set to 0.0299 , then $z_{0}$ is determined only by $H_{s}$ through Eq. (4.4); using the Carter (1982) relations in the range 2-20 s for $T_{p}$, we obtain $z_{0}$ increasing with $H_{s}$ and equal to $0.0031 \mathrm{~m}$ for $T_{p}=20 \mathrm{~s}$, which corresponds to $U_{10}=27.4 \mathrm{~m} \mathrm{~s}^{-1}$. However, it is unlikely to have a full spectrum generated by this wind value. A full JONSWAP spectrum generated by $U_{10}=15 \mathrm{~m} \mathrm{~s}^{-1}$ has $T_{p}=10.9 \mathrm{~s}, H_{s}=5.6 \mathrm{~m}$, and $L_{p}=187 \mathrm{~m}$ in deep water; in this case, the WaSt parameterization provides the roughness length $z_{0}=0.00093 \mathrm{~m}$. Also, a $z_{0}$ can be related to a large range of steepness if the spectrum is not fully developed. For these reasons, it seems more instructive to evaluate the impact of WaSt as $z_{0}$ varies.

Figure 1 exhibits $U_{10}$ after the new equilibrium is achieved with $z_{0}$ ranging from 0.0001 to $0.0009 \mathrm{~m}$. In general, we note a great impact, even for small $z_{0}$. For example, for $z_{0}=0.0005 \mathrm{~m}, U_{10}$ is reduced to $12.9 \mathrm{~m} \mathrm{~s}^{-1}$. The explanation for this result is that, since $z_{0}$ is fixed for each experiment, the only way to accommodate the changes of $u_{*}$ and Obukhov length equilibrium is by the adjustment of $U_{10}$. 


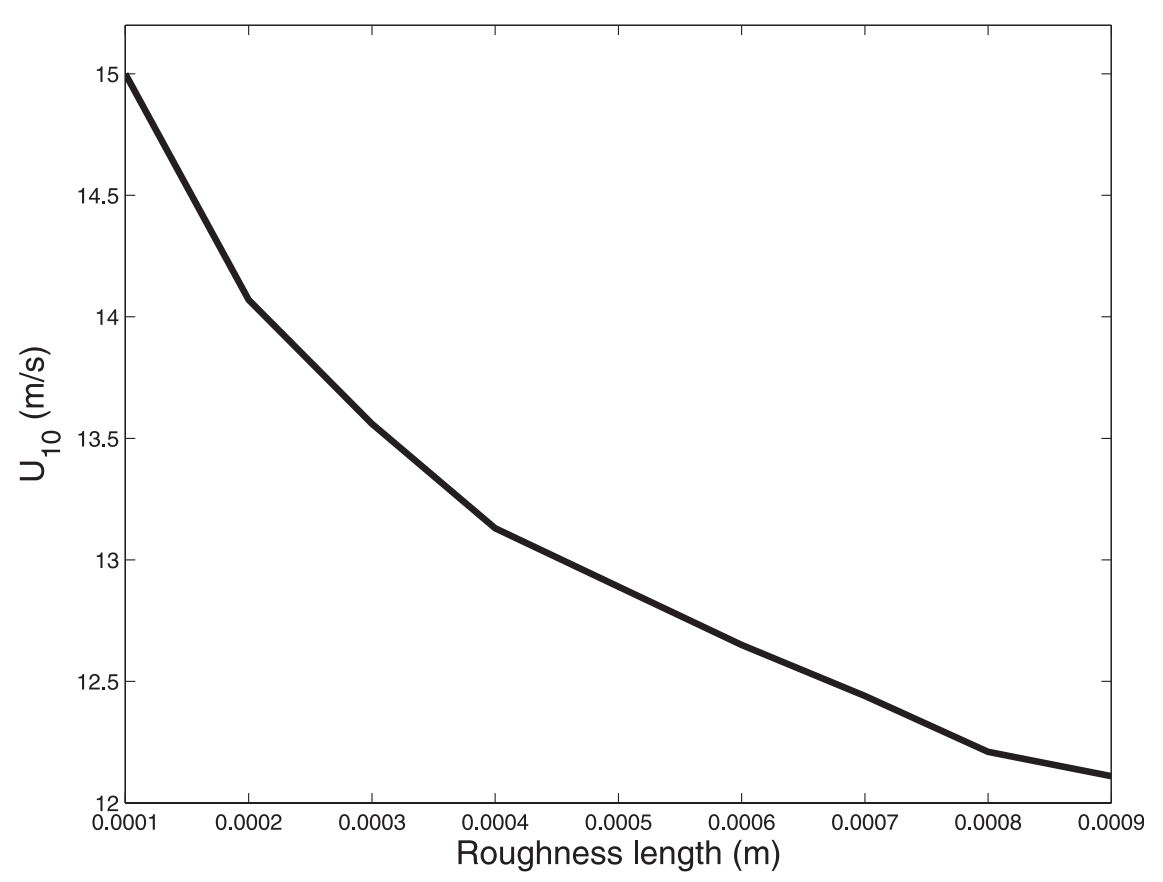

FIG. 1. Steady-state speed $U_{10}\left(\mathrm{~m} \mathrm{~s}^{-1}\right)$ as a function of the roughness length $z_{0}(\mathrm{~m})$ using the Taylor surface stress parameterization. The initial atmosphere has $U_{10}=15 \mathrm{~m} \mathrm{~s}^{-1}$, potential temperature $280 \mathrm{~K}$, and relative humidity $90 \%$, except at surface where the wind speed is zero and the temperature is $285 \mathrm{~K}$. The sea temperature and salinity are $285 \mathrm{~K}$ and 0.034 , respectively. The surface pressure is $1013.5 \mathrm{hPa}$. Heat and mass transported by droplets are switched off.

\section{2) VOLKOV PARAMETERIZATION (WAAG)}

Although in the WaAg parameterization the forcing incoming wave is specified by $C_{p}$ constant, in contrast to the WaSt parameterization $z_{0}$ can change responding to $u_{*}$. Therefore, during a numerical simulation with $C_{p}$ fixed, the scale wave age can evolve until a new wind profile in equilibrium with the imposed wave is established. Then, to quantify the effect of this parameterization, it seems better to study the impact on the model profile resulting from perturbation owing to spectra represented by $T_{p}$ (or $C_{p}$ ). We will obtain its role by varying $T_{p}$ from 2 to $16 \mathrm{~s}$, which corresponds to a fully developed spectrum generated by $U_{10}$ from 2.7 to $22 \mathrm{~m} \mathrm{~s}^{-1}$ in deep water.

Figure 2 shows $z_{0}$ and $U_{10}$ after a steady state is established. One can note that the maximum impact on $U_{10}$ reduces it to $11.9 \mathrm{~m} \mathrm{~s}^{-1}$ when $z_{0}$ is about $0.002 \mathrm{~m}$ for $T_{p}=2 \mathrm{~s}$. For $T_{p} \geq 11 \mathrm{~s}$, the impact on $U_{10}$ is minimal, less than $3 \%$. In contrast with $\mathrm{WaAg}$, the WaSt parameterization shows that the greater $T_{p}$ is, the larger the impact on the wind profile. The reason for the difference is that the $\mathrm{WaAg}$ parameterization uses a parameter describing the maturity of the wave age: since the experiment starts with a $10-\mathrm{m}$ wind $15 \mathrm{~m} \mathrm{~s}^{-1}$, which corresponds to $T_{p}=10.9 \mathrm{~s}$ [according to Carter's (1982) relations], any spectrum with $T_{p}$ smaller is considered young; in this situation, the $\mathrm{WaAg}$ parameterization responds with large $\alpha$ [Eq. (4.3)] and $z_{0}$ is enhanced. Then $u_{*}$ increases while $U_{10}$ decreases. In the ensuing time steps, $U_{10}$ decreases owing to the strong wave stress, reducing $z_{0}$ and $u_{*}$ to establish a new equilibrium. Actually, this parameterization transfers more energy from the wind when the spectrum is young. The older the spectrum, the smaller the $\alpha$, the $z_{0}$ and the impact on the wind.

\section{3) JANSSEN PARAMETERIZATION (WIAC)}

In experiments with the WiAc parameterization, we perturbed the initial condition imposing a fully developed spectrum with $T_{p}$ varying from 2 to $20 \mathrm{~s}$. The results are presented in Fig. 3 with a solid line. One can note the impact on $U_{10}$ increasing with $T_{p}$ up to $6 \mathrm{~s}$ and remaining nearly constant beyond $8 \mathrm{~s}$. This means that low frequencies have smaller impact on the results. To confirm this assessment, we carry out simulations with the same range of $T_{p}$, but imposing (i) no energy at frequencies higher than the first model frequency corresponding to $T=2 \mathrm{~s}$ (dashed line in Fig. 3) and (ii) no energy at frequencies smaller than the first model frequency (dotted-dashed line). The results show nearly no impact on the wind profile when the low frequencies are 


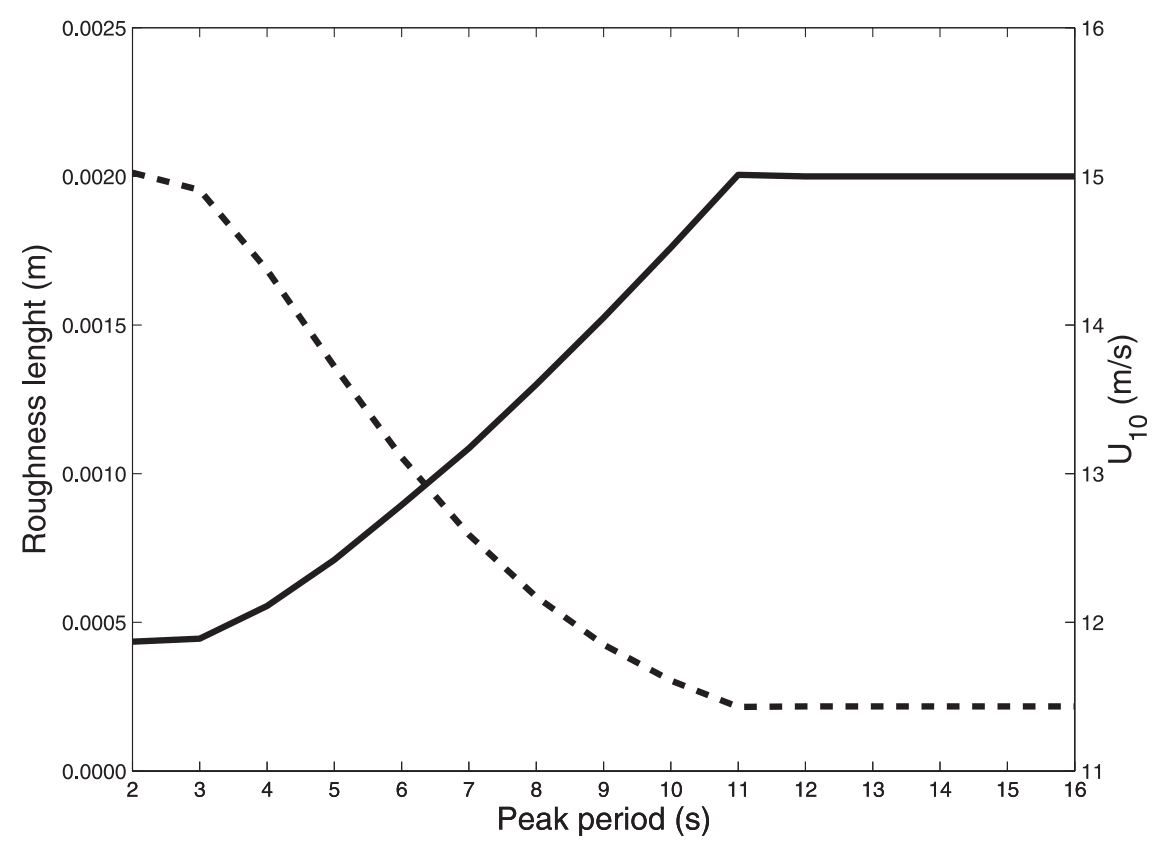

FIG. 2. Roughness length $z_{0}(\mathrm{~m})$ (dashed line with vertical axis in the left) and speed $U_{10}\left(\mathrm{~m} \mathrm{~s}^{-1}\right.$ ) (solid line with vertical axis in the right) at equilibrium as a function of the peak period $T_{p}(\mathrm{~s})$, using the Volkov surface stress parameterization: initial conditions as in Fig. 1.

discharged. On the other hand, the high frequencies still have a great impact on the profile. As opposed to the WaSt and WaAg parameterizations, the WiAc parameterization does not feel any scale, it simply transfers energy to the wave, and this depends strongly on the highfrequency stage of the spectrum: the higher the energy transference by wind onto high frequencies, the more pronounced the impact.

\section{b. Droplet load stress}

The wave-induced stress and droplet load certainly are implicitly considered in the WaAg and WaSt parameterizations, as both are obtained from observations. However, in a numerical model with the analytical WiAc parameterization these effects must be incorporated explicitly. As presented in section 2c, the surface stress depends on $\tau_{\text {wave }}$, whose effect was studied above, and $\tau_{\text {spray }}$, given by Eq. (3.6). The latter stress is computed with $u_{*}$ and $f_{p}$, both required by the windsea Reynolds number, whereas $H_{s}$ is used to calculate $u_{\text {spray }}$ with Eq. (3.5). The experiments with the WiAc parameterization described above are carried out with $T_{p}$ from 2 to $20 \mathrm{~s}$, but including $\tau_{\text {spray }}$ provided by the respective fully developed spectrum and imposing initially $U_{10}=15$ or $30 \mathrm{~m} \mathrm{~s}^{-1}$. The results are presented in Fig. 4, where the dashed (solid) line represents the steady wind when the load spray is included (excluded); as should be expected, the greater the period, the more noticeable the load effect. However, it is very small for $U_{10}=15 \mathrm{~m} \mathrm{~s}^{-1}$ but increases with $U_{10}$; for example, for $T_{p}=20 \mathrm{~s}$, the reduction without (with) load is from 30 to $24.7(23.8) \mathrm{m} \mathrm{s}^{-1}$.

\section{c. Latent and sensible heat}

To study the effect of latent and sensible heat, the initial profile in equilibrium is perturbed by a fully developed wave spectrum with peak period ranging from 2 to $20 \mathrm{~s}$. Then the model is integrated until a new steady state is achieved. Since the sea temperature is $5 \mathrm{~K}$ higher, we expect a positive contribution to the air temperature due to sensible heat. The negative contribution owing to the droplet evaporation will depend on the air relative humidity. We conduct the experiments with initial relative humidity equal to $50 \%, 60 \%, 70 \%, 80 \%, 90 \%, 95 \%$, and $100 \%$; the equilibrium temperature $\theta_{10}$ at $z=10 \mathrm{~m}$ obtained is plotted in Fig. 5 as a function of $T_{p}$.

The results with $R_{\%}=100 \%$ show $\theta_{10}$ increasing. As $T_{p}$ increases, the amount of injected warmer sea mass is higher; therefore, the sensible heat transfer is higher. However, even in this case there is some cooling by evaporation because, when the air temperature increases due to the sensible heat, the relative humidity decreases, then some water will be evaporated. In the experiment with $R_{\%}=95 \%$, the air shows a smaller heating for all $T_{p}$ analyzed, certainly owing to the evaporative cooling effect.

Figure 5 displays an interesting feature around $T_{p}=$ $10 \mathrm{~s}$, where a maximum cooling is observed. A closer analysis of the results reveals that, with increased $T_{p}$, the amount of sea mass ejected increases; therefore, so does 


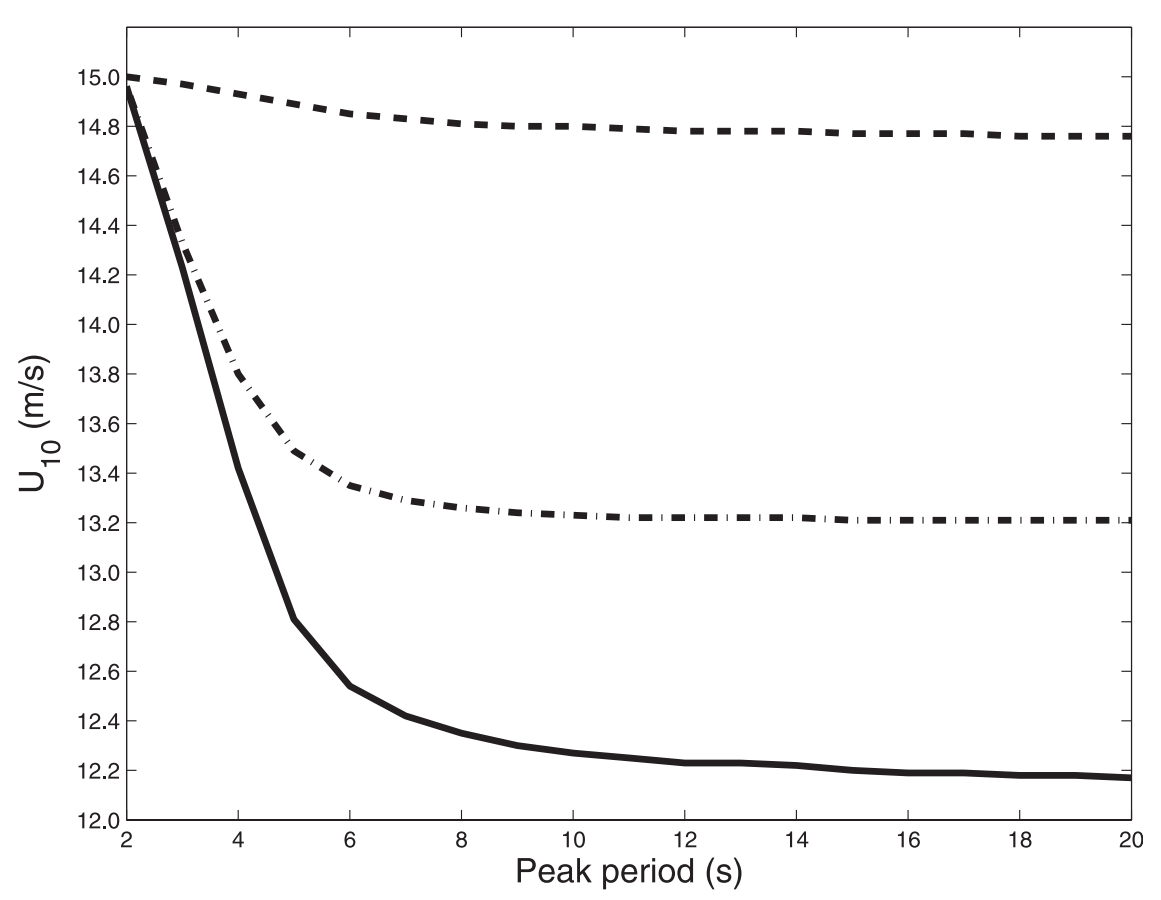

FIG. 3. Steady-state speed $U_{10}\left(\mathrm{~m} \mathrm{~s}^{-1}\right)$ as a function of the peak period $T_{p}(\mathrm{~s})$, using the Janssen surface stress parameterization. The solid line is for the complete wave spectrum, the dotted-dashed line for spectrum comprising frequencies corresponding to periods smaller than $2 \mathrm{~s}$, and the dotted line for periods greater than 2 s: initial conditions as in Fig. 1.

the water available for evaporation. However, unlike the sensible heating, the evaporation is not proportional to the ejected mass; for example, when $R \% \approx 100 \%$, almost all ejected droplets return to the sea and there is nearly no evaporation. Because the sensible heat always increases with $T_{p}$ but the evaporation remains nearly constant from a limiting $T_{p}$, the cooling becomes less significant for high $T_{p}$; therefore, the consequence is an increase in the air temperature. This can be noted in Fig. 5 for $T_{p}>10 \mathrm{~s}$.

\section{Summary and conclusions}

We have investigated the response of an atmospheric boundary layer model to parameterizations of the effect of a sea surface wave spectrum. The model is one dimensional, and the initial profiles of momentum, temperature, and mixing ratio are maintained in steady state by an external forcing. A surface wave spectrum disturbs the balance through surface stress and spume droplets ejected into the atmosphere by breaking waves. The model is integrated until new steady profiles within the boundary layer are obtained, so the effect of the wave spectrum and the parameterization employed can be evaluated. The imposed wave field is not allowed to be changed by the local wind during the integration. This approach is reasonable because the atmosphere's response to surface changes is immediate, whereas the wave spectrum can take hours or days to achieve the fully developed stage.

The effect of the wave stress is simulated and compared using three quite different approaches: wave age (WaAg) (Volkov 2001), wave steepness (WaSt) (Taylor and Yelland 2001), and wind action on the wave spectrum (WiAc) (Janssen 1991) parameterizations. While WiAc uses the complete wave spectrum to compute the stress, WaAg and WaSt are scaled with parameters derived from it-namely, wavelength and speed of the peak frequency and significant wave height (respectively, $L_{p}$, $C_{p}$, and $H_{s}$ ). The experiments are carried out with a constant wind $U_{10}=15 \mathrm{~m} \mathrm{~s}^{-1}$, and each perturbation is constituted by a full developed spectrum characterized by its peak period varying from 2 to $20 \mathrm{~s}$.

The results show the maximum roughness length $z_{0}=$ $0.002 \mathrm{~m}$ in WaAg for $T_{p}=2 \mathrm{~s}$, reducing $U_{10}$ to $11.9 \mathrm{~m} \mathrm{~s}^{-1}$. For a $10-\mathrm{m}$ wind of $15 \mathrm{~m} \mathrm{~s}^{-1}$ a spectrum with $T_{p}=2 \mathrm{~s}$ is in its young stage, the wave age parameterization provides a large $z_{0}$ so as to reduce the wind and transfers momentum to the wave until a new equilibrium is achieved; because in our model the wave spectrum is not allowed to grow, the only possible effect is a reduction of the wind. For old waves $\mathrm{WaAg}$ provides a small $z_{0}$, then the waves have a negligible effect. In contrast with $\mathrm{WaAg}$, the WaSt parameterization uses only $H_{s}$ and $L_{p}$ and no information about the wind speed; it provides a high $z_{0}$ if $H_{s}$ is large, 


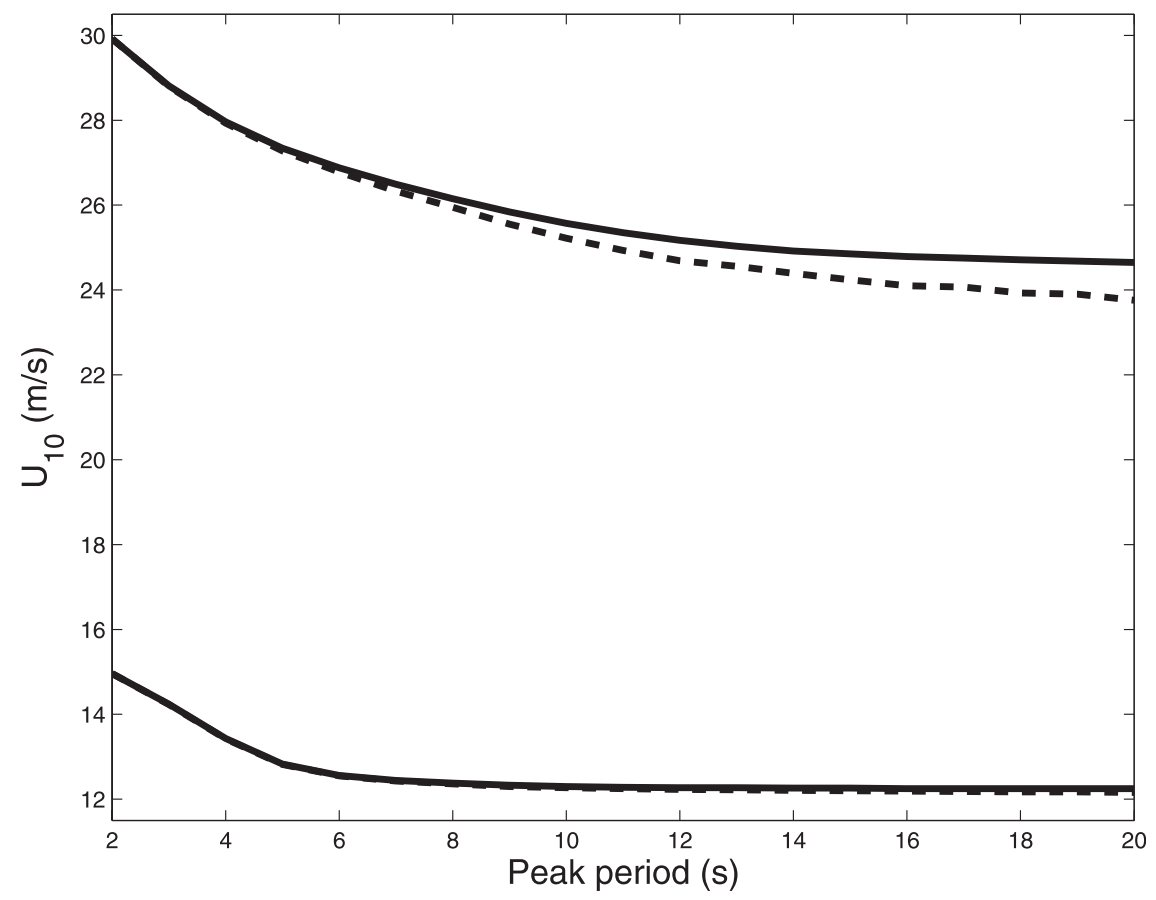

FIG. 4. As in Fig. 3, but with initial $U_{10}=15$ and $30 \mathrm{~m} \mathrm{~s}^{-1}$ with (without) droplet load represented by solid line (dashed line).

which is the case for a fully developed spectrum with an enhanced $T_{p}$. So, the effect of WaSt parameterization in our experiments is opposite to that of $\mathrm{WaAg}$.

On the other hand, the WiAc parameterization is very sensitive to how the energy is distributed among the frequencies, resulting in a large surface stress when there is energy at high frequencies. The model shows that spectral components with periods greater than $2.5 \mathrm{~s}$ have nearly no effect on the wind profile. Also the droplet load contribution to the total surface stress computed by this parameterization can be neglected for wind $U_{10}=$ $15 \mathrm{~m} \mathrm{~s}^{-1}$. However, when the wind speed is doubled, the stress due to the droplet load becomes nearly $1 / 5$ of the total stress.

Observations reveal that most of the wave stress arises at high frequencies, which is truly simulated only by the WiAc parameterization. Less accurate, the WaAg is sensitive to young waves and can be employed to parameterize the wave effect, but in this aspect the WaSt parameterization cannot be implemented because it does not feel the high frequency waves.

In addition to the study of the surface stress effect on the wind profile, the heat transfer accompanying the production of spume droplets is examined with a microphysical model designed to be implemented in operational weather forecasting models. It is similar to the simplified droplet microphysics presented by Andreas (2005), where the droplet evolution is described by four parameters: equilibrium radius, equilibrium temperature, and their respective $e$-folding times. In the Andreas model these parameters are predicted as functions of air temperature $T_{a}$, ambient relative humidity $R \%$, initial radius $r_{i}$, and sea salinity $S$; with the droplet residence time computed from $H_{s}$, the sensible and latent heat fluxes are calculated for each droplet. Then, the integral over the radii [or over only one representative radius, as suggested by Andreas et al. (2008) and Zhang et al. (2006)] estimates these quantities, which are used to compute the heat flux at the top of the droplet evaporation layer (DEL). A boundary layer model transmits the fluxes to higher levels.

The approximation suggested here to account for the effect of the heat released differs from Andreas (2005) in the following aspects:

- Only droplets with radius from 30 to $500 \mu \mathrm{m}$ are considered.

- The sensible heat is immediately released, so the $e$-folding time for the equilibrium temperature is not necessary.

- Each droplet evolves toward a final radius according to its equilibrium radius and residence time while the air temperature decreases, but the overall droplet effect does not lead to a supersaturated layer.

- The sea salinity variation has small impact for radii superior to $30 \mu \mathrm{m}$ and is considered constant. 


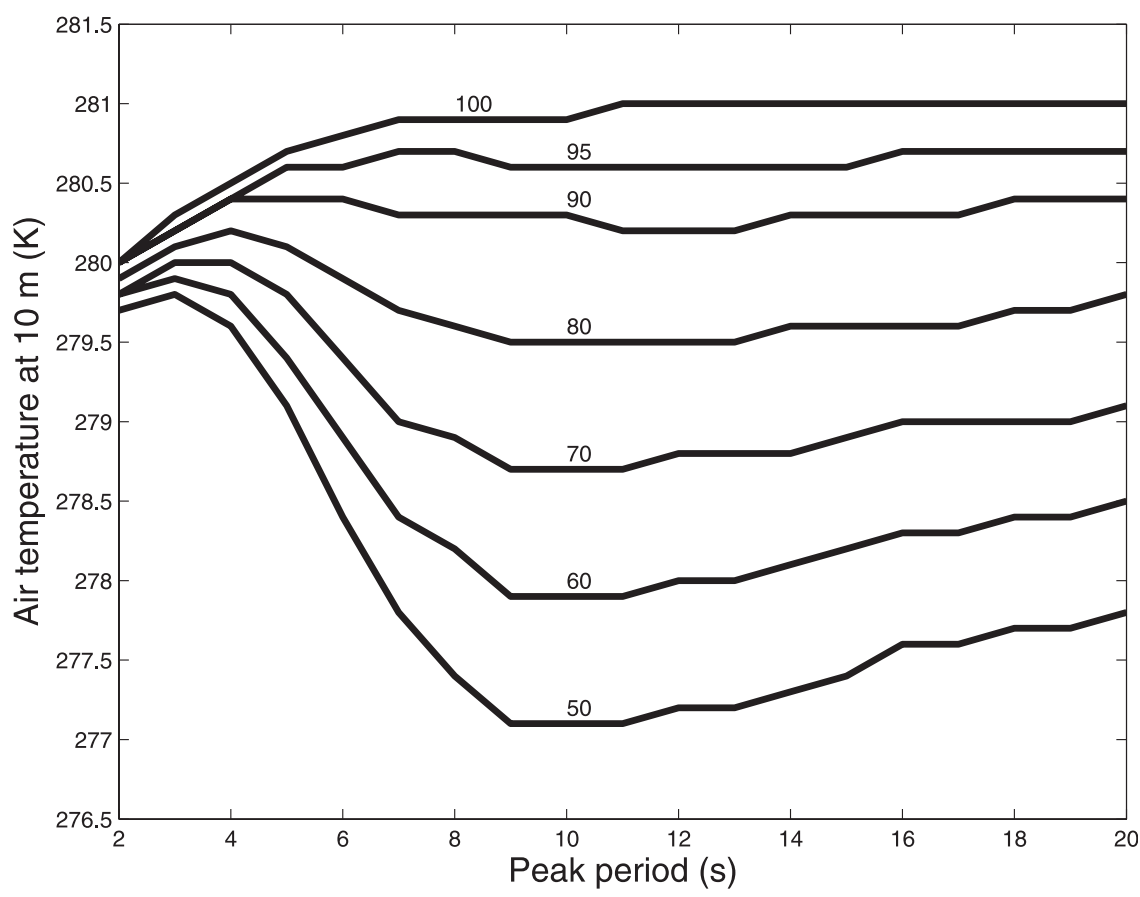

FIG. 5. Temperature at $z=10 \mathrm{~m}$ as a function of the peak period $T_{p}$ (s) for an initial air relative humidity of $100 \%, 95 \%, 90 \%, 80 \%, 70 \%, 60 \%$, and $50 \%$, using the Janssen surface stress parameterization. The sensible and latent heat release effects due to the droplet spume spectrum are included. Initial conditions as in Fig. 1.

- The droplet spectrum is assumed as a product of two functions, so the integral over the droplet radii can be analytically evaluated and stored in a table, depending on $T_{a}, R_{\%}$, and $H_{s}$. The computation of the latent heat is completed by multiplying the table value with the windsea Reynolds number.

- Instead of predicting the heat fluxes at the top of the DEL, the heat and water vapor released are distributed into two sublayers within the first model slice (from surface to $10 \mathrm{~m}$ ), and the partition depends on $H_{s}$ such that when it is smaller than $1 \mathrm{~m}$ only the sublayer adjacent to the surface is affected.

Concerning the last assumption, the distribution of heat release and water vapor into the two sublayers is based on qualitative arguments with magnitudes depending on $H_{s}$ : thus the higher the wave, the more homogeneous the partition.

The effects of these approaches are investigated imposing sea and air surface temperature $285 \mathrm{~K}$, air temperature $280 \mathrm{~K}$ at higher levels, and wind speed $15 \mathrm{~m} \mathrm{~s}^{-1}$. Therefore, the sensible heat warms the atmosphere while the latent heat cools it. The balance between these two opposite effects is controlled by (i) the wave spectrum, which is responsible for the amount of water mass launched into the atmosphere transporting sensible heat, and (ii) the air relative humidity, which controls the latent heat. As should be expected, for $R_{\%}=100 \%$ the cooling is small and the air temperature increases. Also, the smaller the $R_{\%}$, the more pronounced the cooling. The dominance of the cooling is noticeable mainly in the range of smaller significant wave height; for example, the air temperature decreases $3 \mathrm{~K}$ for $R_{\%}=50 \%$ and $H_{s}=$ $4.6 \mathrm{~m}$ (corresponding to $T_{p}=10 \mathrm{~s}$ for a fully developed spectrum): for such low $R_{\%}$, the warming effect increases with increasing $H_{s}$ so that, for $H_{s}>5 \mathrm{~m}$, the cooling becomes less competitive and the warming more noticeable.

For future research, we plan to implement the WiAc surface stress and the droplet parameterization proposed in an operational atmospheric model coupled with a sea surface wave model so as to simulate realistic synoptic-scale meteorological events accompanied by strong wind, such as in developing extratropical cyclones, and calm wind with large fetch, as observed in the trade wind region.

Acknowledgments. We are grateful for comments, suggestions, and constructive criticism from an anonymous reviewer and from Dr. Edgar L Andreas. The comments lead to the inclusion of clarifying discussion and improvements in the material presented. We thank Dr. Peter Caplan for his comments and suggestions for 
improving the English version of this manuscript. This research was supported by Fundação de Amparo à Pesquisa do Estado de São Paulo (FAPESP) Grants 2005/ 59438-9 and 2008/58595-1.

\section{REFERENCES}

Andreas, E. L, 1992: Sea spray and the turbulent air sea heat fluxes. J. Geophys. Res., 97, 11 429-11 441.

_ 1998: A new sea spray generation function for wind speeds up to $32 \mathrm{~m} \mathrm{~s}^{-1}$. J. Phys. Oceanogr., 28, 2175-2184.

- 2002: A review of the sea spray generation function for the open ocean. Atmosphere-Ocean Interactions, Vol. 1, W. Perrie, Ed., WIT Press, 1-46.

—_, 2004: Spray stress revisited. J. Phys. Oceanogr., 34, 1429-1440. , 2005: Approximation formulas for the microphysical properties of saline droplets. Atmos. Res., 75, 323-345.

— 2009: A new value of the von Kármán constant: Implications and implementation. J. Appl. Meteor. Climatol., 48, 923-944.

_- and K. Emanuel, 2001: Effect of sea spray on tropical cyclone intensity. J. Atmos. Sci., 58, 3741-3751.

— , P. O. G. Persson, and J. E. Hare, 2008: A bulk turbulent airsea flux algorithm for high-wind, spray conditions. J. Phys. Oceanogr., 38, 1581-1596.

Businger, J. A., J. C. Wyngaard, Y. Izumi, and E. F. Bradley, 1971: Flux-profile relationships in the atmospheric surface layer. J. Atmos. Sci., 28, 181-189.

Carter, D. J. T., 1982: Prediction of wave height and period for a constant wind velocity using the JONSWAP results. Ocean Eng., 9, 17-33.

Cavaleri, L., and P. Malanotte-Rizzoli, 1981: Wind wave prediction in shallow water: Theory and application. J. Geophys. Res., 86, 10 961-10 974.

Charnock, H., 1955: Wind stress on a water surface. Quart. J. Roy. Meteor. Soc., 81, 639-640.

Donelan, M. A., 1998: Air-water exchange processes. Physical Processes in Lakes and Oceans, J. Imberger, Ed., Vol. 54, Coastal and Estuarine Studies Series, Amer. Geophys. Union, 19-36.

Drennan, W. M., P. K. Taylor, and M. J. Yelland, 2005: Parameterizing the sea surface roughness. J. Phys. Oceanogr., 35, 835-848.

Fairall, C. W., J. D. Kepert, and G. J. Holland, 1994: The effect of sea spray on surface energy transport over the ocean. Global Atmos. Ocean Syst., 2, 121-142.

Friedlander, S. K., 1977: Smoke, Dust, and Haze: Fundamentals of Aerosol Behavior. John Wiley and Sons, 367 pp.

Holthuijsen, L. H., 2007: Waves in Oceanic and Coastal Waters. Cambridge University Press, 387 pp.

Iida, N., Y. Toba, and M. Chaen, 1992: A new expression for the production rate of sea water droplets on the sea surface. J. Oceanogr., 48, 439-460.

Innocentini, V., and E. S. C. Neto, 1992: A numerical study of the role of humidity in the updraft driven by moist slantwise convection. J. Atmos. Sci., 49, 1092-1106.

Janssen, P. A. E. M., 1989: Wave-induced stress and drag of air flow over sea waves. J. Phys. Oceanogr., 19, 745-754.

_- 1991: Quasi-linear theory of wind wave generation applied to wave forecasting. J. Phys. Oceanogr., 21, 1631-1642.

_ 1992: Experimental evidence of the effect of surface waves on the airflow. J. Phys. Oceanogr., 22, 1600-1604.

Krishnamurti, T. N., V. Wong, H.-L. Pan, R. Pasch, J. Molinari, and P. Ardanuy, 1983: A three-dimensional planetary boundary layer model for the Somali jet. J. Atmos. Sci., 40, 894-908.
Ling, S. C., T. W. Kao, and A. I. Saad, 1980: Microdroplets and transport of moisture from ocean. J. Eng. Mech. Div., 106, $1327-1339$

Liu, W. T., K. B. Katsaros, and J. A. Businger, 1979: Bulk parameterizations of air-sea exchanges of heat and water vapor including the molecular constraints at the interface. J. Atmos. Sci., 36, 1722-1735.

Makin, V. K., and V. N. Kudryavtsev, 1999: Coupled sea surfaceatmosphere model. Part 1: Wind over waves coupling. J. Geophys. Res., 104, 7613-7623.

- — - and C. Mastenbroek, 1995: Drag of the sea surface. Bound.-Layer Meteor., 73, 159-182.

Miles, J. W., 1957: On the generation of surface waves by shear flows. J. Fluid Mech., 3, 185-204.

Monahan, E. C., D. E. Spiel, and K. L. Davidson, 1986: A model of marine aerosol generation via whitecaps and wave disruption. Oceanic Whitecaps, E. C. Manahan, Ed., D. Reidel, 167-174.

Monin, A. S., and A. M. Obukhov, 1954: Basic laws of turbulent mixing in the ground layer of the atmosphere. Tr. Geofiz. Inst. Akad. Nauk SSSR, 151, 163-187.

Phillips, O. M., 1957: On the generation of wave by turbulence wind. J. Fluid Mech., 2, 417-445.

Pielke, R. A., 2002: Mesoscale Meteorological Modeling. Academic Press, 676 pp.

Plant, W. J., 2009: The ocean wave height variance spectrum: Wavenumber peak versus frequency peak. J. Phys. Oceanogr., 39, 2382-2383.

Pruppacher, H. R., and J. D. Klett, 1978: Microphysics of Clouds and Precipitation. D. Reidel, 714 pp.

Smith, S. D., and Coauthors, 1992: Sea surface wind stress and drag coefficients: The HEXOS results. Bound.-Layer Meteor., 60, $109-142$.

Soong, S.-T., and Y. Ogura, 1973: A comparison between axisymmetric and slab-symmetric cumulus cloud models. J. Atmos. Sci., 30, 879-893.

Sverdrup, H. U., and W. H. Munk, 1947: Wind, sea, and swell: Theory of relations for forecasting. Hydrographic Office Publication 601, 44 pp.

Taylor, P. K., and M. J. Yelland, 2001: The dependence of sea surface roughness on the height and steepness of the waves. J. Phys. Oceanogr., 31, 572-590.

Toba, Y., S. Komori, Y. Suzuki, and D. Zhao, 2006: Similarity and dissimilarity in air sea momentum and $\mathrm{CO}_{2}$ transfer: The nondimensional transfer coefficients in light of the windsea Reynolds number. Atmosphere-Ocean Interactions, Vol. 2, W. Perrie, Ed., WIT Press, 53-82.

Volkov, Y., 2001: The dependence on wave age. Wind Stress over the Ocean, I. S. F. Jones and Y. Toba, Eds., Cambridge University, 206-217.

Wu, J., 1973: Spray in the atmosphere surface layer: Laboratory study. J. Geophys. Res., 78, 511-519.

, 1998: Insignificant evaporation from escaping sea spray droplets. J. Geophys. Res., 103, 3163-3165.

Zeng, X., M. Zhao, and R. E. Dickinson, 1998: Intercomparison of bulk aerodynamic algorithms for sea surface fluxes using the TOGA COARE and TAO data. J. Climate, 11, 2628-2644.

Zhang, W., W. Perrie, and W. Li, 2006: Impacts of waves and sea spray on midlatitude storm structure and intensity. Mon. Wea. Rev., 134, 2418-2442.

Zhao, D., Y. Toba, K. Sugioka, and S. Komori, 2006: New sea spray generation function for spume droplets. J. Geophys. Res., 111, $1-11$. 